\title{
Initial Factors Influencing Duration of Hospital Stay in Adult Patients With Peritonsillar Abscess
}

\author{
Yu-Hsi Liu' $\cdot$ Hsing-Hao Su${ }^{1} \cdot$ Yi-Wen Tsai ${ }^{2}$ Yu-Yi Hou ${ }^{1} \cdot$ Kuo-Ping Chang ${ }^{1} \cdot$ Chao-Chuan Chi' $\cdot$ Ming-Yee Lin ${ }^{1}$ \\ $\mathrm{Pi}-\mathrm{H}$ siung $\mathrm{Wu}^{1}$
}

Departments of ${ }^{1}$ Otorhinolaryngology-Head and Neck Surgery and ${ }^{2}$ Medical Education and Research, Kaohsiung Veterans General Hospital, Kaohsiung, Taiwan

Objectives. To review cases of peritonsillar abscess and investigate the initial clinical factors that may influence the duration of hospitalization. To determine the predictive factors of prolonged hospital stay in adult patients with peritonsillar abscess.

Methods. Subjects were adults hospitalized with peritonsillar abscess. We retrospectively reviewed 377 medical records from 1990 to 2013 in a tertiary medical center in southern Taiwan. The association between clinical characteristics and the length of hospital stay was analyzed with independent $t$-test, univariate linear regression and multiple linear regression analysis.

Results. The mean duration of hospitalization was $6.2 \pm 6.0$ days. With univariate linear regression, a prolonged hospital stay was associated with several variables, including female gender, older ages, nonsmoking status, diabetes mellitus, hypertension, band forms in white blood cell (WBC) counts, and lower hemoglobin levels. With multiple linear regression analysis, four independent predictors of hospital stay were noted: years of age $(P<0.001)$, history of diabetes mellitus $(P<0.001)$, ratio of band form WBC $(P<0.001)$, and hemoglobin levels $(P<0.001)$.

Conclusion. In adult patients with peritonsillar abscess, older ages, history of diabetes mellitus, band forms in WBC counts and lower hemoglobin levels were independent predictors of longer hospitalization.

Keywords. Diabetes Mellitus; Hemoglobins; Length of Stay; Peritonsillar Abscess

\section{INTRODUCTION}

Peritonsillar abscess (PTA), also known as "quinsy," is the most common complication of acute tonsillitis [1]. It often originates from acute viral tonsillitis with secondary bacterial infection or by other pathogenic mechanism such as obstruction of the supratonsillar Weber's glands [2]. PTA is a localized deep neck infection either within the capsules or with extension to adjacent structures, and progression of the infectious process may cause

\footnotetext{
- Received November 3,2015

Revised January 5, 2016

Accepted March 11, 2016

- Corresponding author: Hsing-Hao Su

Department of Otorhinolaryngology-Head and Neck Surgery, Kaohsiung

Veterans General Hospital, No. 386, Dazhong 1st Road., Zuoying Dist.,

Kaohsiung 81362, Taiwan R.O.C

Tel: +886-7-342-2121, Fax: +886-7-346-8223

E-mail: shsu@vghks.gov.tw
}

septicemia, aspiration of the contents of a ruptured abscess, or airway obstruction, or may even cause rupture of the great vessels [3].

PTAs are often polymicrobial [4]. In PTA, predominant bacterial species may differ as a result of underlying conditions [5]. Antibiotics administered with supportive care may help in uncomplicated cases; however, adequate drainage via aspiration or surgery is recognized as an appropriate treatment strategy [6]. Admission may be required for adequate management. To our best knowledge, there is little literature focusing on the initial factors that may prolong the hospital stay of PTA. It is worth noting that the same factors may also influence the severity, morbidity, and financial cost of the disease. We analyzed records of our hospitalized patients during the past two decades, to identify the initial factors affecting the duration of hospitalization of patients with PTA.

Copyright (C) 2017 by Korean Society of Otorhinolaryngology-Head and Neck Surgery.

This is an open-access article distributed under the terms of the Creative Commons Attribution Non-Commercial License (http://creativecommons.org/licenses/by-nc/4.0)

which permits unrestricted non-commercial use, distribution, and reproduction in any medium, provided the original work is properly cited. 


\section{MATERIALS AND METHODS}

\section{Patients}

This retrospective study included 377 cases of PTA in patients admitted to a tertiary medical center from June 1990 to June 2013. Inclusion criteria were: diagnosis of PTA by clinical examination confirmed by aspiration of pus or by computed tomography (CT) of the head and neck. Children less than 18 years of age, and adults, who were re-admitted for postoperative complications or disease sequelae, were excluded from this study. The study was approved by the Institutional Review Board of Kaohsiung Veterans General Hospital.

\section{Study design}

At the first review of records, gender, age, body mass index and factors including medication, smoking, alcoholism, and betel nut chewing were documented. Initial physical findings were recorded as well. Needle aspiration was performed for sampling and drainage of pus over the suspected area, and samples for bacterial culture, and blood for laboratory examination were collected. Imaging surveys including chest plain film and head and neck CT were arranged if a large volume of pus was present or if the patient had relatively severe symptoms.

Patients may have required admission for further procedures or parental antibiotics, or may have been followed in the clinics for oral medications. Incision and drainage, quinsy tonsillectomy, or exploratory cervical fasciotomy may have been performed if the initial parental antibiotics had been insufficient. The decision of discharge was depended on the patient's condition. Most patients were discharged when their conditions became stable for 3 days after using systemic antibiotics or abscess drainage. If there was any complication happened, the hospital stay would be extended till the problem was solved.

We reviewed the medical records of each patient, and documented and analyzed demographics, comorbidities, substance use/abuse, laboratory blood results, bacteriology, therapeutic strategy, duration of hospitalization and complications.

\section{Statistics}

The descriptive data are presented as mean \pm standard deviation. The analysis is undertaken in two parts. Independent $t$-test and univariate linear regression analysis are utilized to determine

\section{H I G G L I G G H T/S}

- Predictive factors of prolonged hospital stay were examined in 377 adult patients with peritonsillar abscess.

- Host factors related with longer hospitalization: older age, diabetes.

- Initial data showed poor prognosis: high ratio of band form white blood cell, low hemoglobin level. the relationship between each variable and the length of hospital stay. The categorized data is analyzed with independent $t$ test, while the continuous variable was calculated with linear regression analysis. The $P$-value less than 0.05 is considered of statistical significance. Those variables with $P$-value $<0.05$ in univariate analysis are evaluated by multiple linear regression analysis with the enter method. It is to test the independence of these predictors and the strength of their contribution to hospital stay. Analysis is performed using SPSS ver. 20 (IBM Co., Armonk, NY, USA).

\section{RESULTS}

\section{Demographics}

Four hundred and fifteen patients with PTA were admitted to the tertiary medical center during our study period; however, 38 of these patients were younger than 18 years old and were excluded from this study. Among 377 adult patients, 269 male and 108 female patients were enrolled. Mean age was $43.2 \pm$ 16.8 years old (range, 18 to 85 years; median age, 41 years). Fifty patients $(13.3 \%)$ were older than 65 years of age. Most patients were between 21 and 50 years of age (Table 1).

Mean duration of hospitalization was $6.2 \pm 6.0$ days (range, 1 to 65 days; median, 5.0 days) for all patients, $5.7 \pm 4.2$ days in the age group between 18-65 years of age and $9.8 \pm 12.5$ days in the group of older than 65 years of age. Eight patients $(2.1 \%)$ were readmitted due to contralateral PTA. Eight patients (2.1\%) had sequelae of diseases or postoperative complications.

\section{Diabetes mellitus}

Forty patients had past medical history of diabetes mellitus $(10.6 \%)$.Ten patients were older than 65 years of age $(2.7 \%)$.

\section{Cigarette smoking and alcohol and betel nut comsumption}

One hundred and seventy-four patients smoked cigarettes (46.2\%), 12 of whom were older than 65 years of age (3.2\%). One hundred and twenty-six patients drank alcohol (33.4\%), 11 of whom were older than 65 years of age $(2.9 \%)$. Fifty-nine

Table 1. Demographics of patients by age groups

\begin{tabular}{lccc}
\hline \multirow{2}{*}{ Variable } & \multicolumn{2}{c}{ Age group (yr) } & \multirow{2}{*}{ Total } \\
\cline { 2 - 3 } & $18-65$ & $>65$ & \\
\hline Case & $327(86.2)$ & $50(13.8)$ & $377(100)$ \\
Sex & & & \\
$\quad$ Female & $90(23.9)$ & $18(4.8)$ & $108(28.6)$ \\
$\quad$ Male & $237(62.9)$ & $32(8.5)$ & $269(71.4)$ \\
Diabetes mellitus & $30(8.0)$ & $10(2.7)$ & $40(10.6)$ \\
Cigarette smoking & $162(43.0)$ & $12(3.2)$ & $174(46.2)$ \\
Alcoholism & $115(30.5)$ & $11(2.9)$ & $126(33.4)$ \\
Betel nut chewing & $55(14.6)$ & $4(1.1)$ & $59(15.6)$ \\
\hline
\end{tabular}

Values are presented as number (\%). 
patients chewed betel nuts $(15.6 \%)$, four of whom were older than 65 years of age $(1.1 \%)$.

\section{Laboratory surveillance}

We routinely checked complete blood cell counts, differential cell counts and blood biochemistry including electrolytes, liver function tests, and the blood glucose level for all patients. Among them, 277 patients had leukocytosis with a white blood cell (WBC) count $>11,000(73.5 \%)$, and 63 patients had bandform WBCs (16.7\%). C-reactive protein levels (CRP) were measured in 142 patients, and elevated CRP was detected in 125 of them (>1.0 gm/dL, 88.0\%).

\section{Microbiology and antibiotic treatment}

Results of pus cultures either from surgery or from needle aspiration were available for 255 patients. Among them, 212 patients $(83.1 \%)$ had bacterial growth from these cultures. One hundred and twenty-three bacterial cultures (58.0\%) were reported to be polymicrobial.

The most common pathogens identified through pus culture was Streptococcus viridans (42 of 212, 19.8\%), followed by Klebsiella pneumonia (39 of 212, 18.4\%). The most of the anaerobes was Prevotella (24 of 212, 11.3\%).

\section{Methods for pus drainage}

Blind fine needle aspiration was performed at the suspected abscess site, not only for culture but also for drainage. Once PTA was suspected, CT of head and neck was performed for evaluating the range of abscess involvement, and other possible deep neck infections.

Antibiotic treatment only was the initial treatment when only peritonsillar cellulitis was detected, or when only a small amount of pus was aspired. If residual PTA was present, immediate or secondary quinsy tonsillectomy was performed; however, exploratory cervical fasciotomy of the neck was considered if other deep neck abscess was detected by imaging studies. Accordingly, 276 patients $(73.2 \%)$ underwent quinsy tonsillectomy, 10 patients $(2.7 \%)$ underwent exploratory cervical fasciotomy, $14(3.7 \%)$ experienced resolution of symptoms with multiple needle aspirations, and $81(21.5 \%)$ completed treatment with medication alone.

\section{Complications}

In all 377 cases with or without surgical intervention, few complications were noted. Initially, three patients $(0.8 \%)$ had sepsis, and five patients $(1.3 \%)$ had airway obstruction and were intubated or underwent tracheostomy. Eight patients (2.1\%) experienced tonsillar bed bleeding after quinsy tonsillectomy, and one patient $(0.3 \%)$ had a prolonged pharyngo-cutaneous fistula. There was no severe complication including internal jugular thrombosis, carotid erosion with hemorrhage, intracranial infection, or death.

\section{Factors associated with hospital stay}

The length of stay in patients with female gender, history of hypertension and elevated segmentedWBC counts showed statistically significant by univariate analysis $(P<0.05)$. Moreover, older ages, histories of diabetes mellitus and hypertension, positive band form WBCs, and lower hemoglobin levels showed a strongly statistically significant increase in duration of hospitalization $(P<0.01)$. Smokers had negative effects about hospital stays than nonsmokers $(P<0.01)$. There was no statistical significance between the groups with or without pus drainage respectively (Table 2).

Table 2. Different variables affecting the hospital stay were analyzed with univariate linear regression

\begin{tabular}{|c|c|c|}
\hline Variable & Hospital stay (day, mean \pm SD) & $P$-value \\
\hline Sex & & 0.031 \\
\hline Male & $5.7 \pm 5.6$ & \\
\hline Female & $7.4 \pm 7.1$ & \\
\hline Age (yr) & & $<0.001$ \\
\hline$>65$ & $9.8 \pm 12.5$ & \\
\hline$\leq 65$ & $5.7 \pm 4.2$ & \\
\hline Smoking & & $<0.001$ \\
\hline Yes & $5.1 \pm 2.6$ & \\
\hline No & $7.3 \pm 7.9$ & \\
\hline Alcoholism & & 0.243 \\
\hline Yes & $5.7 \pm 5.1$ & \\
\hline No & $6.5 \pm 6.6$ & \\
\hline Betel nut chewing & & 0.091 \\
\hline Yes & $5.0 \pm 2.5$ & \\
\hline No & $6.5 \pm 6.6$ & \\
\hline Diabetes mellitus & & 0.008 \\
\hline Yes & $11.1 \pm 12.3$ & \\
\hline No & $5.6 \pm 4.6$ & \\
\hline Hypertension & & 0.027 \\
\hline Yes & $8.9 \pm 10.1$ & \\
\hline No & $5.8 \pm 5.0$ & \\
\hline WBC $\left(\times 10^{3} / \mathrm{mL}\right)$ & & 0.131 \\
\hline$>12$ & $6.5 \pm 6.9$ & \\
\hline$\leq 12$ & $5.6 \pm 4.2$ & \\
\hline Band form WBC & & 0.007 \\
\hline Yes & $10.1 \pm 12.7$ & \\
\hline No & $5.5 \pm 3.3$ & \\
\hline Segmented WBC (\%) & & 0.011 \\
\hline$\geq 80$ & $7.3 \pm 8.1$ & \\
\hline$<80$ & $5.5 \pm 4.0$ & \\
\hline Hemoglobin (g/dL) & & 0.007 \\
\hline$\geq 13$ & $5.7 \pm 3.9$ & \\
\hline$<13$ & $10.2 \pm 12.0$ & \\
\hline C-reactive protein & & 0.657 \\
\hline$\geq 4$ & $6.3 \pm 5.6$ & \\
\hline$<4$ & $5.8 \pm 6.4$ & \\
\hline Pus drainage & & 0.247 \\
\hline Yes & $6.0 \pm 6.0$ & \\
\hline No & $6.8 \pm 6.2$ & \\
\hline
\end{tabular}

SD, standard deviation; WBC, white blood cell. 
Table 3. Predictors of length of hospital stay, evaluated by multiple linear regression analysis

\begin{tabular}{lccc}
\hline Variable & Regression coefficient (B) & Standard error & $P$-value \\
\hline Age $(\mathrm{yr})$ & 0.078 & 0.019 & $<0.001$ \\
Diabetes mellitus & 4.389 & 1.009 & $<0.001$ \\
$\begin{array}{l}\text { Band form white } \\
\text { blood cell }(\%)\end{array}$ & 0.425 & 0.114 & $<0.001$ \\
Hemoglobin $(\mathrm{g} / \mathrm{dL})$ & -0.720 & 0.199 & $<0.001$ \\
\hline
\end{tabular}

Constant $(B)=12.435$, standard error $=3.069$.

Variables with statistical significance in univariate analysis were tested by multiple linear regression with the enter method. Four independent factors contributing to the length of stay were: years of age $(P<0.001)$, history of diabetes mellitus $(P<0.001)$, ratio of band form WBCs $(P<0.001)$ and hemoglobin level $(P<0.001)$ (Table 3$)$.

\section{DISCUSSION}

PTA is a common aerodigestive tract suppurative infection that may be life-threatening and require long-term treatment. Admission was indicated for hydration, parenteral antibiotics, and adequate drainage of pus.

We analyzed the medical records of patients admitted for PTA in the past two decades. Though there were several clinical variables associated with longer hospital stay, four independent predictors were identified via multivariate linear regression: years of age, history of diabetes mellitus, ratio of band form $\mathrm{WBC}$ and hemoglobin level. Our findings indicate the patients with special characteristics may have prolonged hospital stay. Such characteristics as laboratory data are exclusively found in medical records, not in the database of health insurance. They are useful for the practitioners who stand in the first line. These findings would alert clinicians to treat such risky patients more carefully. These independent factors in our series are discussed with literature review.

\section{Age}

Most persons in this cohort were 21 to 50 years of age, similar to the age distribution of patients described in several recent reports [7-9]. Older patients had a higher prevalence of associated systemic diseases, less-effective immunological response, and less-complete tissue recovery, and might need longer observation and medical management. In the series of Marioni et al. [10] with 103 patients, $23 \%$ of patients were older than 65 years of age, and had longer hospital stay and complication rates, similar to the results in our study. Marom et al. [7] analyzed 427 patients, of whom $24.4 \%$ patients were older than 40 years of age. They had longer hospitalization and more complications than the group of younger than 40 years of age. Also, a trend of increased incidence rate of PTA in elderly was observed in the study. Qureshi et al. [11] investigated nationwide inpatient sample, and found that age groups of 40 to 64 years, 65 and older were associated with increased risk of retropharyngeal abscess. In our series, there was a tendency for older patients to have longer duration of hospitalization. Thus, age served as an independent predictor in our linear regression model.

\section{Band form in white blood cell}

Immature WBCs known as band form cells are released from the bone marrow into the peripheral blood in the presence of severe infection. Band form cell counts are routinely obtained in patients with infectious disease, and may be used to evaluate the severity of sepsis [12]. However, it is controversial whether band counts may be useful in predicting inpatient mortality [13]. In the current study, band form WBC could be found in about $16 \%$ of all patients, and there was a trend in these patients to have longer hospitalizations.

\section{Diabetes mellitus}

Patients with diabetes mellitus are considered to be immunocompromised, and have higher infection rates. Elevated blood sugar level $\geq 200 \mathrm{gm} / \mathrm{dL}$ is associated with reduced neutrophil activity [14]. In deep neck infections, diabetes mellitus is the most common systemic comorbidity [15-18]. A more severe infectious process, and higher complication and mortality rates are associated with diabetes mellitus $[15,19,20]$. It is also associated with infections that are more severe, as well as a higher rate of complications, requiring a longer time for treatment and observation. Moreover, infection status and poor oral nutrition during the course of the disease may worsen control of blood glucose. Studies have shown an increased prevalence of diabetes mellitus from the year $2000(5.8 \%)$ to $2007(8.3 \%)$ in Taiwan. In our study, $9.7 \%$ of all patients had diabetes. They had a longer hospitalization, similar to the result that Huang et al. [20] demonstrated in diabetic patients with deep neck infections. However, it was limited that we confirmed the comorbidity of diabetes mellitus but the controlling status was not evaluated objectively.

\section{Hemoglobin level}

The hemoglobin level is related to iron metabolism, especially under inflammatory conditions. Anemia of inflammation occurs during infections with a microbial pathogen, including bacterial, viral, or yeast infections [21]. Changes in iron stores, erythrocyte production, and degradation all contribute to inflammationrelated anemia. With increasing severity of the infectious process, a lower hemoglobin level can be detected.

Several mechanisms were addressed in the relationship between infection/inflammation and anemia, including iron sequestration and the role of hepcidin [21]. Iron sequestration plays an important role in the antimicrobial systems of hosts, which might inhibit the proliferation of invading pathogens [22]. 
Hepcidin is an iron-regulatory hormone. Modulation of hepcidin expression during infection decreases the availability of iron to invading pathogens. This response also restricts the iron that is available to erythropoietic precursors, and might contribute to the anemia associated with infections and inflammatory disorders [23].

In our cohort, serum iron and hepcidin were not checked routinely, but the hemoglobin level was measured. The severity of the infectious process could be reflected by the length of hospitalization. In our linear regression model, decreased hemoglobin level was identified as an independent predictor for longer hospital stay.

\section{Miscellaneous}

Cigarette smoking, alcohol and betel nut consumption may damage the oral mucosa, thus increasing permeability to carcinogens and pathogenic microorganisms, thereby increasing the risk of infectious disease and malignancy of the oral cavity and oropharynx [9,24]. Smoking is considered to be a predisposing factor for PTA [9]. Interestingly, we noticed our patients who smoked cigaretts or chewed betel nuts had shorter duration of hospitalization by independent $t$-tests; however, further analysis showed no statistical significance between these dangerous habits and prolonged hospital stay in our study.

Elevated CRP was thought to be associated with inflammatory status. Liu et al. [25] reported a retrospective study of 240 patients undergoing incision and drainage because of a deep neck infection. Persisted postoperative discharge was correlated with the elevated preoperative CRP level [25]. In our series, only 142 patients had CRP test. And there was no statistical significance between CRP and length of hospital stay.

The choice of surgical intervention was controversial in patients with PTA. Adequate surgical drainage was necessary when abscess formation was confirmed, and is followed with antibiotic treatments. There are many reviews supporting different methods for pus drainage, including fine needle aspiration, incision and drainage, immediate tonsillectomy, and interval tonsillectomy $[3,4,6,8]$. In our adult PTA patients, there was no significant shortening of hospital stay through these pus-drainage procedures.

In conclusion, in adult patients with PTA, older ages, positive band form WBC, lower hemoglobin levels and having medical history of diabetes mellitus were independent predictors for longer duration of hospitalization.

\section{CONFLICT OF INTEREST}

No potential conflict of interest relevant to this article was reported.

\section{ACKNOWLEDGMENTS}

The authors thank Professor Hsueh-Wen Chang (Department of Biological Sciences, National Sun Yat-sen University, Kaohsiung, Taiwan) for his help in the statistical analysis.

\section{REFERENCES}

1. Del Mar CB, Glasziou PP, Spinks AB. Antibiotics for sore throat. Cochrane Database Syst Rev. 2006 Oct:(4):CD000023.

2. Passy V. Pathogenesis of peritonsillar abscess. Laryngoscope. 1994 Feb;104(2):185-90.

3. Powell J, Wilson JA. An evidence-based review of peritonsillar abscess. Clin Otolaryngol. 2012 Apr;37(2):136-45.

4. Brook I. Microbiology and management of peritonsillar, retropharyngeal, and parapharyngeal abscesses. J Oral Maxillofac Surg. 2004 Dec;62(12):1545-50.

5. Klug TE. Incidence and microbiology of peritonsillar abscess: the influence of season, age, and gender. Eur J Clin Microbiol Infect Dis. $2014 \mathrm{Jul} ; 33(7): 1163-7$.

6. Herzon FS, Martin AD. Medical and surgical treatment of peritonsillar, retropharyngeal, and parapharyngeal abscesses. Curr Infect Dis Rep. 2006 May;8(3):196-202.

7. Marom T, Cinamon U, Itskoviz D, Roth Y. Changing trends of peritonsillar abscess. Am J Otolaryngol. 2010 May-Jun;31(3):162-7.

8. Steyer TE. Peritonsillar abscess: diagnosis and treatment. Am Fam Physician. 2002 Jan;65(1):93-6.

9. Dunn N, Lane D, Everitt H, Little P. Use of antibiotics for sore throat and incidence of quinsy. Br J Gen Pract. 2007 Jan;57(534):45-9.

10. Marioni G, Castegnaro E, Staffieri C, Rinaldi R, Giacomelli L, Boninsegna $\mathrm{M}$, et al. Deep neck infection in elderly patients: a single institution experience (2000-2004). Aging Clin Exp Res. 2006 Apr; 18(2):127-32.

11. Qureshi HA, Ference EH,Tan BK, Chandra RK, Kern RC, Smith SS. National trends in retropharyngeal abscess among adult inpatients with peritonsillar abscess. Otolaryngol Head Neck Surg. 2015 Apr; 152(4):661-6.

12. Drees M, Kanapathippillai N, Zubrow MT. Bandemia with normal white blood cell counts associated with infection. Am J Med. 2012 Nov;125(11):1124.e9-15.

13. Ward MJ, Fertel BS, Bonomo JB, Smith CL, Hart KW, Lindsell CJ, et al. The degree of bandemia in septic ED patients does not predict inpatient mortality. Am J Emerg Med. 2012 Jan;30(1):181-3.

14. Kawahito S, Kitahata H, Oshita S. Problems associated with glucose toxicity: role of hyperglycemia-induced oxidative stress. World J Gastroenterol. 2009 Sep;15(33):4137-42.

15. Huang TT, LiuTC, Chen PR, Tseng FY, Yeh TH, Chen YS. Deep neck infection: analysis of 185 cases. Head Neck. 2004 Oct;26(10):85460.

16. Parhiscar A, Har-El G. Deep neck abscess: a retrospective review of 210 cases. Ann Otol Rhinol Laryngol. 2001 Nov;110(11):1051-4.

17. Brook I. The role of anaerobic bacteria in upper respiratory tract and other head and neck infections. Curr Infect Dis Rep. 2007 May; 9(3):208-17.

18. Daramola OO, Flanagan CE, Maisel RH, Odland RM. Diagnosis and treatment of deep neck space abscesses. Otolaryngol Head Neck Surg. 2009 Jul;141(1):123-30.

19. Lin HT, Tsai CS, Chen YL, Liang JG. Influence of diabetes mellitus on deep neck infection. J Laryngol Otol. 2006 Aug;120(8):650-4.

20. Huang TT, Tseng FY, Liu TC, Hsu CJ, Chen YS. Deep neck infection in diabetic patients: comparison of clinical picture and outcomes 
with nondiabetic patients. Otolaryngol Head Neck Surg. 2005 Jun; 132(6):943-7.

21. Bullen JJ, Rogers HJ, Spalding PB, Ward CG. Iron and infection: the heart of the matter. FEMS Immunol Med Microbiol. 2005 Mar; 43(3):325-30.

22. Roy CN. Anemia of inflammation. Hematology Am Soc Hematol Educ Program. 2010;2010:276-80.

23. Ganz T. Hepcidin and iron regulation, 10 years later. Blood. 2011 Apr;117(17):4425-33.
24. Wen CP, Tsai MK, Chung WS, Hsu HL, Chang YC, Chan HT, et al. Cancer risks from betel quid chewing beyond oral cancer: a multiple-site carcinogen when acting with smoking. Cancer Causes Control. 2010 Sep;21(9):1427-35.

25. Liu SA, Liang MT, Wang CP, Wang CC, Lin WD, Ho HC, et al. Preoperative blood sugar and $\mathrm{C}$-reactive protein associated with persistent discharge after incision and drainage for patients with deep neck abscesses. Clin Otolaryngol. 2009 Aug;34(4):336-42. 